\title{
Figura/Fundo: Decifrar o Código McLuhan
}

\section{Robert K. Logan}

\section{Resumo}

Neste trabalho, investigamos o uso por McLuhan de figura/fundo, que cumpre um papel central em seu entendimento das mídias e ajuda-nos a decifrar 0 código McLuhan.

\section{Palavras chave}

McLuhan. Figura/Fundo.

\section{Introdução}

Marshall McLuhan é considerado pioneiro no desenvolvimento de estudos da comunicação e fundador do movimento de ecologia das mídias. Há quem ache sua escrita difícil de ler. 0 objetivo do presente texto é decifrar o código McLuhan demonstrando como seu uso de figura/fundo foi central em sua abordagem para entender as mídias e seus efeitos.

A maneira como McLuhan explicava por que as pessoas achavam sua escrita difícil de entender é revelada na seguinte carta a um amigo.

"Meus textos muitas vezes confundem as pessoas porque começo pelo fundo e elas começam pela figura." Começo pelos e trabalho até chegar às , ao passo que o padrão convencional é começar por uma seleção um tanto arbitrária de 'causas' e depois tentar ligá-las a alguns dos efeitos. É esse processo desordenado de emparelhamento que leva à superficialidade fragmentária. Quanto a mim, não tenho um ponto de vista, mas simplesmente trabalho com a situação total como óbvias contra um oculto.

Uma vez que se entenda que o fundo oculto de nosso tempo é a informação viajando à velocidade da luz, torna-se fácil ver por que a escolaridade está mudando tão drasticamente (MOLINARO; MCLUHAN; TOYE, 1987, p. 478). 
McLuhan faz uso de sua noção de figura/ fundo nas passagens acima. Trata-se de um conceito chave em seu trabalho. 0 autor acreditava que, para entender 0 significado de uma figura, é preciso levar em conta o fundo no qual esta funciona e contra o qual está situada. 0 verdadeiro significado de qualquer "figura" - seja esta uma pessoa, um movimento social, um tecnologia, uma instituição, um evento de comunicação, um texto ou um corpo de ideias - só pode ser determinado levandose em conta o fundo ou entorno no qual essa figura funciona. 0 fundo proporciona 0 contexto do qual emerge o pleno significado ou importância de uma figura. A preocupação com a relação figura/fundo é coerente com a ênfase que McLuhan dá a interface e padrão, não a um ponto de vista fixo. Isto também explica por que 0 autor pensava que o conteúdo não era independente do meio no qual era transmitido. 0 meio forma um fundo para 0 conteúdo que transmite e, como tal, modifica a mensagem; esta é mais uma razão por que McLuhan afirmava que o meio é a mensagem. A mensagem de um meio, independente de seu conteúdo, é o fundo que ele cria para qualquer conteúdo que transmite. Assim, o meio de fato possui duas mensagens: uma é figura, ou seu conteúdo, e o outro é o fundo, o fundo que 0 meio cria para seu conteúdo.

Este é um dos exemplos favoritos de McLuhan para ilustrar a maneira como o contexto ou fundo pode transformar o significado de uma figura: a figura de uma chaminé soltando fumaça, outrora símbolo de progresso industrial, especialmente na iconografia soviética, hoje é símbolo de poluição. Outro exemplo é a mudança no significado do bronzeado; no passado era sinal de trabalho árduo no campo, agora é símbolo de afluência e férias, provavelmente um dia será símbolo de negligência irresponsável com a saúde e 0 risco de câncer de pele.

Acredito que 0 uso que 0 autor faz da análise de figura/fundo permitiu-lhe ver coisas que outros não viram, e também explica sua assombrosa capacidade de "prever" o futuro através do estudo do presente. McLuhan escreveu em carta a Tom Stepp de 28 de março de 1973:

A figura é o que aparece, e o fundo é sempre
subliminar. As mudanças ocorrem no fundo antes
de ocorrer na figura. Podemos projetar tanto figu-
ra quanto fundo como imagens do futuro usando
o fundo como subtrama de padrões subliminares
e pressões e efeitos que, na verdade, vêm antes
das figuras mais ou menos finais para as quais
normalmente dirigimos nosso interesse (MOLI-
NARO; MCLUHAN; TOYE, 1987).

McLuhan acrescenta que isto é fácil para ele porque "dedicou quase todo o seu estudo ao fundo". Para McLuhan, fundo também era 0 ambiente em que a figura funcionava, mas, como sugere McLuhan (1969, p. 30), não se trata de um recipiente: " 0 ambiente é processo, não recipiente. 0 ambiente sempre consegue, de algum modo, ser invisível. Só o conteúdo [também se pode ler figura], o ambiente anterior, é perceptível." Ao contrário dos outros estudiosos 
das mídias, que se concentravam na figura, 0 foco do estudo de McLuhan sempre foi o fundo ou ambiente e esta, acredito, era sua vantagem secreta. Também é a forma de entender as muitas dicotomias que 0 autor elaborou em seus estudos.

\section{A Inversão de Causa e Efeito}

"A forma de estudar os efeitos se você quisesse estudar, por exemplo, o que foi o carro a motor; poderia obter mais dados a partir do que ele fez ao meio ambiente e à comunidade." (MCLUHAN; MCLUHAN; STAINES, 2003, p. 90).

McLuhan sugere que a melhor maneira de estudar as mídias era "fazendo inventários de efeitos". (MCLUHAN; MCLUHAN; STAINES, 2003, p. 90). Eu sugeriria, portanto, que a melhor maneira de dar continuidade ao trabalho de McLuhan é fazer um inventário de toda a tecnologia que surgiu desde seu falecimento, em 1980; esta data marca o início da era dos computadores pessoais e da que chamamos popularmente de era digital. Do ponto de vista técnico, os computadores mainframe faziam uso de tecnologia digital. No entanto, apenas uma estreita faixa de especialistas podia acessá-los, e os mainframes eram usados, em grande medida, como forma de mídia de massas; por isto, eu assinalaria 0 advento do computador pessoal, o PC, como a ruptura que marca a fronteira entre a era elétrica da mídia de massas e a era digital de hoje.
Estreitamente ligada ao uso por McLuhan da relação figura/fundo estava sua inversão de causa e efeito, em que 0 autor começava com 0 efeito e retrocedia até a causa que gerara 0 efeito. $\mathrm{Na}$ citação que abre a seção anterior sobre figura/ fundo, McLuhan aponta que começa pelo fundo, ao passo que outros começam pela figura, e também que ele começa pelos efeitos e vai trabalhando até as causas. 0 autor associa claramente os efeitos com o fundo e as causas com a figura. Portanto, sua inversão de causa e efeito está nitidamente relacionada com sua metodologia de figura/fundo. McLuhan também foi influenciado pelo trabalho de artistas, inventores e cientistas. 0 método dos cientistas é observar efeitos e, por experimentação e raciocínio, determinar as causas dos efeitos observados. McLuhan (1964, p. 68) também via o processo criativo, tanto do inventor quanto do artista, como um trabalho que retrocede do efeito que desejam criar até a causa que levará ao efeito desejado.

\section{A Nêmesis da criatividade...}

A. N. Whitehead... explicou que a grande descoberta do século XIX foi a descoberta da técnica da descoberta. A saber, a técnica de começar pelas coisas a serem descobertas e retroceder, trabalhando passo a passo, como em uma linha de montagem, até o ponto de onde é necessário começar para chegar ao objeto desejado. Nas artes, isto significava partir do efeito e depois inventar um poema, um quadro ou um edifício que gere precisamente esse efeito e nenhum outro.

McLuhan (1962, p. 328) também foi muitíssimo influenciado por Edgar Allen Poe e os poetas simbolistas ao elaborar sua noção de que os artistas 
trabalham retrocedendo a partir dos efeitos que querem criar, como revela a seguinte passagem da última seção da Galáxia de Gutenberg:

Poe trabalhou com esse método em muitos de seus poemas e contos. Mas isto é óbvio, sobretudo, em sua invenção da história policial em que Dupin, seu detetive, é um artista-esteta que soluciona crimes por um método de percepção artística. A história policial não só é o grande exemplo popular do trabalho por retrocesso do efeito para a causa, mas é também a forma como o leitor envolve-se profundamente como coautor. Também é o caso na poesia simbolista, a realização plena de cujo efeito, de momento a momento, exige que o leitor participe do próprio processo poético.

A ideia de inversão é a chave para decifrar o Código McLuhan. 0 autor trabalhava retrocedendo dos efeitos para suas causas e do fundo, ou ambiente midiático, para a figura de seu conteúdo. Usou esta técnica para entender o futuro. Uma vez escreveu: "Olhamos para o presente através de um espelho retrovisor. Retrocedemos rumo ao futuro." Em vez de concentrar-se na figura do futuro por meio da especulação, estudou cuidadosamente o fundo do futuro, que é feito do passado e do presente.

Disse: "Sempre tive muito cuidado em jamais prever nada que ainda não tivesse acontecido" (MCLUHAN; MCLUHAN; STAINES, 2003, p. 172).

Também considerava que os artistas orientavam a maneira como olhar para o futuro. McLuhan gostava desta observação de Wyndham Lewis: "0 artista está escrevendo uma história detalhada do futuro porque tem consciência do potencial ocioso do presente (MCLUHAN; MCLUHAN; STAINES, 2003, p. 14)." Creio que este sentimento de Wyndham Lewis contribuiu muito para as ideias de McLuhan a respeito da previsão do futuro com base em suas observações do presente.

\section{Serviço/desserviço}

McLuhan falou muitas vezes sobre o serviço e 0 desserviço que a tecnologia presta, como nos dois trechos abaixo, de duas de suas cartas:

\begin{abstract}
Só estou dizendo que qualquer produto ou inovação cria ambientes tanto de serviço quanto de desserviço que remodelam a atitude humana. Esses ambientes de serviço e desserviço são sempre invisíveis até um novo ambiente suplantá-los. (A Jonathan Miller em 22 de abril de 1970 - MOLINARO; MCLUHAN; TOYE 1987, p. 404).
\end{abstract}

Não tenho teoria alguma sobre nada. Faço observações descobrindo contornos, linhas de força e pressões. Satirizo constantemente e minhas hipérboles não são nada comparadas com os eventos a que se referem. Se estudar o simbolismo, você descobrirá que se trata de uma técnica de despojamento na qual as figuras são deliberadamente privadas de seu fundo. Você parece não ter compreendido que a mensagem, em sua relação com o meio, nunca é o conteúdo, mas os efeitos combinados do meio como ambiente de serviço e desserviço (A William Kuhns 6 de dezembro de 1971 - MOLINARO; MCLUHAN; TOYE 1987, p. 448).

Pode-se encarar a identificação de McLuhan do serviço e desserviço das mídias como outro exemplo de seu uso de figura/fundo onde 0 
serviço, que era a intenção do meio, é a figura, e 0 desserviço não intencional é parte do fundo, ou ambiente, que o meio cria. Uma vez mais, McLuhan começa pelo fundo e observa 0 desserviço, ao contrário de outros acadêmicos da área da comunicação, que se concentram quase totalmente no serviço de um meio, ignorando seus desserviços.

0 que também é interessante na segunda carta, escrita a Kuhns, é que McLuhan reforça sua afirmação de que não começa pela teoria, e também admite explicitamente que faz uso de sátira.

\section{Ambiente/antiambiente}

Outro exemplo do uso por McLuhan de figura/ fundo é sua noção de ambiente e antiambiente, onde ambiente, que é o fundo de qualquer nova tecnologia, torna-se figura em relação a um antiambiente, que funciona como fundo para a figura desse ambiente.

Qualquer nova tecnologia, qualquer extensão ou ampliação das faculdades humanas que é materializada, tende a criar um novo ambiente... É no jogo entre 0 antigo e o novo ambientes que se gera uma incomensurável série de problemas e confusões... É útil enfocar todas as artes e ciências como atuando no papel de antiambientes que nos permitem perceber 0 ambiente (MCLUHAN, 1967a).

Em outras palavras, são os artistas e/ou cientistas que, ao criar 0 antiambiente, permitem que percebamos o novo ambiente criado por um novo meio ou tecnologia. Se não fosse por este antiambiente, só veríamos 0 conteúdo do novo meio, e não 0 ambiente que dá suporte ao novo meio e que o novo meio cria, como observou McLuhan (1970, p. 192) em

Cultura é nosso negócio:

Já que, em qualquer situação, 10 por cento dos eventos causam 90 por cento, ignoramos os 10 por cento e ficamos pasmos com os 90 por cento. Sem antiambiente, todos os ambientes são invisíveis. 0 papel do artista é criar antiambientes como meio de percepção e ajuste. A técnica de detetive de Hamlet para lidar com 0 ambiente oculto ao seu redor foi o do artista: 'por mais que eu me apresente sob aspecto extravagante, tal como em futuro é possível que eu venha a comportar-me'... (l, v, 171-72)

Uma das maneiras como McLuhan preferia descrever nossa cegueira a nossos ambientes era apontar que os peixes não têm consciência da água em que nadam. "Uma coisa sobre a qual os peixes não sabem absolutamente nada é a água, já que não têm antiambiente que lhes permita perceber o elemento dentro do qual vivem." (MCLUHAN; FIORE; AGEL, 1968, p. 175).

Talvez precisemos identificar 0 antiambiente do ambiente digital no qual hoje vivemos.

Será 0 artista digital? Ou talvez os escritos de Marshall McLuhan?

\section{Outros usos que McLuhan fazia da Técnica e da Inversão de Figura/Fundo}

Mostramos como McLuhan usou a noção de figura/fundo para elaborar algumas das outras 
ferramentas que utilizou para entender as mídias, inclusive a inversão de causa e efeito, de serviço e desserviço de todas as mídias, e de ambientes e antiambientes. Encontraremos mais de seu pensamento figura/fundo quando entendermos 0 sentido de alguns de seus famosos aforismos. Em "o meio é a mensagem", por exemplo, é a mensagem que é a figura, e o meio, o fundo. 0 mesmo é verdade em relação à expressão "0 usuário é o conteúdo", em que o conteúdo é a figura e o usuário, o fundo. Ele também defendeu a inversão da relação entre produtor e usuário.

Eu gostaria de concluir esta discussão sobre figura/fundo com um par de sondas - o que é uma forma de dizer que vou especular. Como observou McLuhan, o que tinha de único a maneira como abordava 0 entendimento das mídias era usar a análise figura/fundo e enfatizar o fundo, não a figura, os efeitos, não as causas, o desserviço, não 0 serviço, 0 antiambiente, não 0 ambiente, 0 meio, não a mensagem, e o usuário, não 0 conteúdo.

Seu foco em cada um desses pares diádicos foi exatamente oposto ao de outros acadêmicos da área das mídias. Ele mesmo admitiu ter recorrido à hipérbole para fazer-se entender neste ponto porque estava remando contra a corrente habitual nos estudos da comunicação.

A segunda sonda é a minha crença de que foi preciso um pensador altamente letrado para elaborar a abordagem figura/fundo que McLuhan desenvolveu. A noção de figura/fundo com que McLuhan analisa as mídias, inclusive as elétricas, vem de uma perspectiva literária em que figura e fundo podem ser distinguidos. No espaço acústico da comunicação oral e da comunicação eletricamente configurada, não há figura e fundo, mas só um campo. McLuhan está trabalhando a partir da tradição literária, bem ao contrário do que afirmam alguns, a saber, que ele é cativo da comunicação elétrica. $\mathrm{Na}$ verdade, McLuhan estava, a meu ver, tentando preservar a tradição literária e tentando resgatar-nos da invasão insidiosa da cultura televisiva. Felizmente, devido à ubiquidade da informática com computadores pessoais, smart phones e Internet, a obsolescência do texto escrito decorrente da televisão foi revertida, pois o texto agora passa a representar uma porcentagem muito maior do conteúdo das mídias digitais do que nas comunicações dominadas pelas mídias elétricas de massa mídia do tempo de McLuhan. Muitos dos padrões das comunicações elétricas que ele identificou como descentralização, interdisciplinaridade, contração da lacuna entre produtor e consumidor, substituição dos empregos por papéis e conhecimento como motores de nossa economia persistiram com as mídias digitais e, na verdade, acentuaram-se como veremos no capítulo 5 .

Uma das características da perspectiva figura/ fundo é que o que se considerada como figura e fundo pode ser invertido, como em certas imagens criadas por Escher. Em um campo, todos os elementos do campo podem ser vistos como figura, e os demais, como fundo dessa figura. 


\section{Meio é a Mensagem}

"A mensagem de qualquer meio ou tecnologia é a mudança de escala ou ritmo ou padrão que introduz nas coisas humanas." (MCLUHAN, 1964, p.8).

"As sociedades sempre foram plasmadas mais pela natureza dos meios pelos quais os homens se comunicam do que pelo conteúdo da comunicação." (MCLUHAN, 1967b).

"O meio é a mensagem é uma das observações mais úteis proferidas no século XX." (MAILER, 1988, p. 117).

0 significado deste aforismo é que, independente de seu conteúdo ou pretensa mensagem, as mídias têm seus próprios efeitos intrínsecos sobre nossa percepção, que são a mensagem singular dessa mídia. "A mais recente abordagem dos estudos das mídias considera não só o 'conteúdo', mas o meio e a matriz cultural dentro da qual aquele determinado meio funciona." (MCLUHAN, 1964, p. 11). A mensagem de um meio, independente de seu conteúdo, é o fundo que ele cria para qualquer conteúdo que transmite. Assim, o meio de fato transmite duas mensagens: uma é figura, ou seu conteúdo, e o outro é o fundo, o fundo que cria para seu conteúdo. 0 meio é a mensagem é um exemplo clássico de como McLuhan inverte a relação figura/fundo e escolhe concentrar-se no fundo, não na figura.

\section{Usuário é o Conteúdo}

A ideia que 0 aforismo "o usuário é o conteúdo" transmite é mais um exemplo da inversão que McLuhan efetua na ênfase da figura ou conteúdo para 0 fundo ou usuário. 0 conteúdo é a figura e 0 usuário é 0 fundo.

\section{Leis da mídia (LdaM)}

São quatro as Leis da Mídia (LdaM) para 0 estudo dos efeitos contraintuitivos das mídias, tecnologias, leis científicas ou qualquer artefato feito pelo homem:

1. Todo meio, tecnologia ou artefato feito pelo homem acentua alguma função humana.

2. Ao fazê-lo, causa a obsolescência de algum meio, tecnologia ou artefato anteriormente feito pelo homem, que antes era usado para cumprir a mesma função.

3. Ao cumprir sua função, o novo meio, tecnologia ou artefato feito pelo homem recupera alguma forma mais antiga do passado.

4. E, quando levado longe 0 bastante, o novo meio, tecnologia ou artefato feito pelo homem se inverte, dá uma reviravolta, tornando-se uma forma complementar ou possivelmente oposta.

As Leis da Mídia (LdaM) são outro exemplo do uso que McLuhan faz da relação figura/fundo. 
0 meio que amplia alguma função humana e é tema da primeira lei é a figura. 0 meio que sofre obsolescência e o meio que é recuperado são 0 fundo. E o novo meio que o meio da primeira lei se torna é a nova figura. Portanto, as LdaM têm duas figuras e dois fundos.

\section{Um Replay de Figura/Fundo}

"Em todos os padrões, quando o fundo muda, a figura também é alterada pela nova interface" (MCLUHAN; MCLUHAN; STAINES, 2003, p. 180).

"Vivemos na era do replay instantâneo, e este é um dos mais notáveis fatos de qualquer era, pois permite que tenhamos o significado sem a experiência... 0 replay é uma técnica não de cognição, mas de reconhecimento." (MCLUHAN; MCLUHAN; STAINES, 2003, p.218-219).

Creio que, de todas as suas várias técnicas, a relação figura/fundo e o foco de McLuhan no fundo, não na figura, constituem a chave para seus muitos insights; isto invariavelmente acarreta a formação de um par de dois elementos, um dos quais é a figura - que percebemos conscientemente e que é alvo da maior parte da atenção de outros acadêmicos - e 0 outro é 0 fundo - de que a maioria não tem consciência, com exceção dos artistas. É no fundo que McLuhan se concentra antes de retroceder invariavelmente para a figura. Examinamos uma série de exemplos do uso que McLuhan faz de figura/fundo, o que, a meu ver, é a chave para entender sua abordagem do entendimento das mídias e, portanto, vai permitir que o leitor decifre o código McLuhan. Em apoio a esta hipótese, apresentarei uma lista de todos os exemplos do uso que McLuhan faz de figura/ fundo, muitos dos quais, mas nem todos, já foram vistos no presente texto.

Começamos por uma citação de seu ensaio de 1972, O fim da ética do trabalho:

0 presente fato é que todos nós vivemos neste novo mundo simultâneo ressonante no qual a relação entre figura e fundo, público e ator, busca de objetivos e desempenho de papéis, centralismo e descentralismo simplesmente mudaram e se inverteram repetidamente (MCLUHAN; MCLUHAN; STAINES, 2003, p. 194).

Na inversão de causa e efeito, é 0 efeito que é 0 fundo e causa, a figura.

Quando se trata de público e ator, é o público que é 0 fundo e 0 ator, a figura.

Na relação entre empregos e papéis, é o papel que é o fundo e o emprego, a figura. "Busca de objetivos" é figura e "desempenho de papéis", fundo.

Considerando ambientes centralizados versus descentralizados, 0 ambiente descentralizado é puro fundo e, no ambiente centralizado, o elemento centralizado é figura e todos os demais elementos ligados à figura centralizadora formam o fundo. 
Quando se trata de perceptos e conceitos, é 0

percepto que é o fundo e o conceito,

a figura.

"Os efeitos são perceptos, e as causas tendem a ser conceitos (MCLUHAN; MCLUHAN; STAINES, 2003, p. 213)."

Em "o meio é a mensagem", é o meio que é 0 fundo e a mensagem, a figura.

Em "o usuário é o conteúdo", é o usuário que é 0 fundo e o conteúdo, a figura.

Em "cada tecnologia tem tanto serviço quanto desserviço", é o desserviço que é o fundo e 0 serviço, a figura.

Em "os produtos tornam-se serviços na era da eletricidade", é o serviço que é o fundo e 0 produto, a figura.

Em "os consumidores tornam-se produtores na era da eletricidade", é o consumidor que é 0 fundo e o produtor, a figura.

Na comparação entre espaço visual e espaço acústico, é o espaço acústico que é o fundo e 0 visual, a figura.

Na comparação entre o meio do mito oral e 0 livro, é o mito oral que é o fundo e o livro, a figura.
Na comparação entre transparência (luz

através) e iluminação (luz sobre), é a

transparência que é o fundo e a iluminação, a figura.

Em "o conteúdo de um novo meio é um meio antigo", é o meio antigo que é o fundo e 0 novo, a figura.

Ao examinarmos os padrões de pensamento do lado direito e do lado esquerdo do cérebro, é 0 lado direito que tem consciência do fundo e 0 esquerdo, da figura.

Ao considerarmos software e hardware, é 0 software que é o fundo e o hardware, a figura.

Ao considerarmos os padrões de pensamento subjetivo e objetivo, é o subjetivo que é o fundo e 0 objetivo, a figura.

Ao considerarmos especialização versus interdisciplinaridade, é a interdisciplinaridade que é o fundo e o especialismo, a figura.

Se considerarmos os monopólios de saber versus o crowdsourcing, é o crowdsourcing que é o fundo e o monopólio de saber, a figura.

Ao contrastarmos um ponto de vista com o reconhecimento de padrão, é 0 reconhecimento de padrão que é o fundo e 0 ponto de vista, a figura. 
Se considerarmos a lógica, é a lógica indutiva ou analógica que é o fundo e a lógica dedutiva, a figura.

McLuhan aplica figura/fundo até ao humor, no qual uma piada é a figura e uma queixa, que provoca a piada, é o fundo.

Ao considerar a política, as políticas dos políticos cumprem o papel de figura e a imagem dos políticos, o de fundo.

"À velocidade da luz, políticas e partidos políticos cedem o lugar a imagens carismáticas."

"A política acabará sendo substituída por imagens. 0 político ficará muito contente por abdicar em favor de sua imagem, porque a imagem será muito mais poderosa do que ele jamais poderia ser."

Ao examinar a educação, as respostas ou informações condensadas cumprem o papel de figura e as perguntas exploratórias, o de fundo.

Em sua análise do jornalismo, o foco do antigo jornalismo objetivo está na figura, ao passo que 0 do novo jornalismo de Normal Mailer e Tom Wolfe, está no fundo.

Ao considerar o programa e seus efeitos subliminares, é o programa que é a figura e os efeitos subliminares, 0 fundo.
Até em sua análise de ambiente e antiambiente, 0 ambiente, que costuma ter papel de fundo e ser oculto, passa a ser figura, ao passo que 0 antiambiente, revelado a nós por artistas e cientistas que nos tornam conscientes do ambiente subliminar, é fundo.

0 par figura/fundo mais extravagante que McLuhan criou talvez tenha sido Sputnik e planeta Terra. "Sputnik... talvez uma extensão do próprio planeta... A natureza acabou... o planeta tornou-se uma forma de arte, um ambiente ecologicamente programável (MCLUHAN; MCLUHAN; STAINES, 2003, p. 208)." Sputnik é a figura e o planeta é o fundo.

Outro par criado por McLuhan é que o conteúdo de um novo meio é algum meio antigo. "Salvo a luz, todos os outros meios vêm em pares, um deles atuando como conteúdo do outro, obscurecendo o funcionamento de ambos. (MCLUHAN;

ZINGRONE, 1995, p. 274)."

Percebo que minha análise desses pares de McLuhan é uma grande generalização, mas acredito que contém um elemento de verdade. Creio que falará a muitos McLuhanitas, e certamente ofenderá os que têm uma visão negativa do pensamento de McLuhan. No entanto, parece-me curioso que McLuhan contraste constantemente esses pares. Esta abordagem está implícita em sua quarta Lei das Mídias, na qual está a reviravolta ou inversão. Esta abordagem talvez se deva, 
em parte, ao fato de 0 autor ter pensado

constantemente em termos de metáfora, que considerava como uma relação figura/fundo.

"Todas as metáforas são figura/fundo em

relação à figura/fundo (MCLUHAN; MCLUHAN; STAINES, 2003, p. 289)."

\section{Referências}

MAILER, Norman. Antigonish Review, 117, p. 74-75, 1988.

MCLUHAN, Eric; ZINGRONE, Frank (Ed.). Essential McLuhan. Concord Ontario: Anansi, 1995.

MCLUHAN, Marshall. The Gutenberg Galaxy: The Making of Typographic Man. Toronto: University of Toronto Press, 1962.

Understanding Media: Extensions of Man.

New York: McGraw Hill, 1964. The page references in the text are for the McGraw Hill paperback second edition. Readers should be aware that the pagination in other editions is different. To aid the reader in calibrating note that Chapter 1 The Medium is the Message begins on page 7 in the edition I have referenced.

The Relation of Environment to Anti-

Environment. University of Windsor Review, v. 11, n.1, p. 1-10, Fall, 1966a. Reprinted in: Matson, F. and Montagu, A. (eds) (1967). The Human Dialogue. New York: Macmillan, 1-10; reprinted in: Moos, M. A. (ed) (1997). Media Research: Technology, Art, Communication: Essays by Marshall McLuhan. Amsterdam: G+B Arts International, 110-20; reprinted in McLuhan, M. (2005). Marshall McLuhan Unbound. Eric McLuhan (ed.), Corte Madera, CA: Gingko Press. Electronics and the Psychic Dropout. This

Magazine Is About Schools, v. 1, n. 1, p. 37-42, $1966 \mathrm{~b}$. The Relation of Environment to AntiEnvironment. In: MATSON, Floyd W.; MONTAGU,
Ashley (Ed.). The Human Dialogue: Perspectives on Communication. New York: Free Press, 1967a. p. 39-47. The New Education. The Basilian Teacher, v. 11, n. 2, p. 66-73, $1967 \mathrm{~b}$.

Playboy Magazine Interview. Playboy

Magazine, Mar. 1969.

Culture is Our Business. New York: MacGraw Hill, 1970.

MCLUHAN, Marshall; FIORE, Quentin; AGEL, Michael. War and Peace in the Global Village. New York: Bantam Books, 1968.

MCLUHAN, Marshall; MCLUHAN, Stephanie; STAINES, David. Understanding Me: Lectures and Interviews.

Toronto: McClelland and Stewart, 2003.

MOLINARO, Matie; MCLUHAN, Corrine; TOYE, William. (Ed.). 1987. Letters of Marshall McLuhan. Toronto: Oxford University Press. 
Figure/Ground: Cracking the McLuhan Code

Figura/fondo: descifrando el código McLuhan

Abstract

In this paper we explore McLuhan's use of figure/ground, which plays a central role in his understanding of media and helps us to crack the McLuhan code.

\section{Keywords}

McLuhan. Figure/ground.

\section{Resumen}

En este trabajo exploramos el uso que hace McLuhan de figura/fondo, que juega un papel central en su comprensión de los medios de comunicación y nos ayuda a descifrar el código McLuhan.

\section{Palabras clave}

McLuhan. Figura/fondo. 


\section{Expediente}

A revista E-Compós é a publicação científica em formato eletrônico da Associação Nacional dos Programas de Pós-Graduação em Comunicação (Compós). Lançada em 2004, tem como principal finalidade difundir a produção acadêmica de pesquisadores da área de Comunicação, inseridos em instituições do Brasil e do exterior.
E-COMPÓS I www.e-compos.org.br I E-ISSN 1808-2599

Revista da Associação Nacional dos Programas

de Pós-Graduação em Comunicação.

Brasília, v.14, n.3, set./dez. 2011.

A identificação das edições, a partir de 2008

passa a ser volume anual com três números.

\section{CONSELHO EDITORIA}

Afonso Albuquerque, Universidade Federal Fluminense, Brasil Alberto Carlos Augusto Klein, Universidade Estadual de Londrina, Brasi Alex Fernando Teixeira Primo, Universidade Federal do Rio Grande do Sul, Brasil Ana Carolina Damboriarena Escosteguy, Pontifícia Universidade Católica do Rio Grande do Sul, Brasil

Ana Gruszynski, Universidade Federal do Rio Grande do Sul, Brasil Ana Silvia Lopes Davi Médola, Universidade Estadual Paulista, Brasil André Luiz Martins Lemos, Universidade Federal da Bahia, Brasil Ângela Freire Prysthon, Universidade Federal de Pernambuco, Brasil Angela Cristina Salgueiro Marques, Faculdade Cásper Líbero (São Paulo), Brasil Antônio Fausto Neto, Universidade do Vale do Rio dos Sinos, Brasil Antonio Carlos Hohlfeldt, Pontifícia Universidade Católica do Rio Grande do Sul, Brasil Antonio Roberto Chiachiri Filho, Faculdade Cásper Líbero, Brasil Arlindo Ribeiro Machado, Universidade de São Paulo, Brasil

Arthur Autran Franco de Sá Neto, Universidade Federal de São Carlos, Brasil Benjamim Picado, Universidade Federal Fluminense, Brasil César Geraldo Guimarães, Universidade Federal de Minas Gerais, Brasi Cristiane Freitas Gutfreind, Pontifícia Universidade Católica do Rio Grande do Sul, Brasi Denilson Lopes, Universidade Federal do Rio de Janeiro, Brasil Denize Correa Araujo, Universidade Tuiuti do Paraná, Brasil

Edilson Cazeloto, Universidade Paulista , Brasi

Eduardo Peñuela Cañizal, Universidade Paulista, Brasi Eduardo Vicente, Universidade de São Paulo, Brasi Eneus Trindade, Universidade de São Paulo, Brasil Erick Felinto de Oliveira, Universidade do Estado do Rio de Janeiro, Brasil Florence Dravet, Universidade Católica de Brasilia, Brasil Francisco Eduardo Menezes Martins, Universidade Tuiuti do Paraná, Brasil Gelson Santana, Universidade Anhembi/Morumbi, Brasil Gilson Vieira Monteiro, Universidade Federal do Amazonas, Brasil Gislene da Silva, Universidade Federal de Santa Catarina, Brasil Guillermo Orozco Gómez, Universidad de Guadalajara Gustavo Daudt Fischer, Universidade do Vale do Rio dos Sinos, Brasil Hector Ospina, Universidad de Manizales, Colômbia Herom Vargas, Universidade Municipal de São Caetano do Sul, Brasil leda Tucherman, Universidade Federal do Rio de Janeiro, Brasi Inês Vitorino, Universidade Federal do Ceará, Brasil Janice Caiafa, Universidade Federal do Rio de Janeiro, Brasi Jay David Bolter, Georgia Institute of Technology Jeder Silveira Janotti Junior, Universidade Federal de Pernambuco, Brasil João Freire Filho, Universidade Federal do Rio de Janeiro, Brasi John DH Downing, University of Texas at Austin, Estados Unido José Afonso da Silva Junior, Universidade Federal de Pernambuco, Brasil
José Carlos Rodrigues, Pontifícia Universidade Católica do Rio de Janeiro, Brasil José Luiz Aidar Prado, Pontifícia Universidade Católica de São Paulo, Brasil José Luiz Warren Jardim Gomes Braga, Universidade do Vale do Rio dos Sinos, Brasi Juremir Machado da Silva, Pontifícia Universidade Católica do Rio Grande do Sul, Brasil Laan Mendes Barros, Universidade Metodista de São Paulo, Brasil Lance Strate, Fordham University, USA, Estados Unidos Lorraine Leu, University of Bristol, Grã-Bretanha Lucia Leão, Pontifícia Universidade Católica de São Paulo, Brasil Luciana Panke, Universidade Federal do Paraná, Brasil Luiz Claudio Martino, Universidade de Brasilia, Brasil Malena Segura Contrera, Universidade Paulista, Brasil Márcio de Vasconcellos Serelle, Pontifícia Universidade Católica de Minas Gerais, Brasil Maria Aparecida Baccega, Universidade de São Paulo e Escola Superior de Propaganda e Marketing, Brasil

Maria das Graças Pinto Coelho, Universidade Federal do Rio Grande do Norte, Brasil Maria Immacolata Vassallo de Lopes, Universidade de São Paulo, Brasil Maria Luiza Martins de Mendonça, Universidade Federal de Goiás, Brasil Mauro de Souza Ventura, Universidade Estadual Paulista, Brasi Mauro Pereira Porto, Tulane University, Estados Unidos Nilda Aparecida Jacks, Universidade Federal do Rio Grande do Sul, Brasil Paulo Roberto Gibaldi Vaz, Universidade Federal do Rio de Janeiro, Brasi Potiguara Mendes Silveira Jr, Universidade Federal de Juiz de Fora, Brasil Renato Cordeiro Gomes, Pontifícia Universidade Católica do Rio de Janeiro, Brasil Robert K Logan, University of Toronto, Canadá

Ronaldo George Helal, Universidade do Estado do Rio de Janeiro, Brasil Rosana de Lima Soares, Universidade de São Paulo, Brasil Rose Melo Rocha, Escola Superior de Propaganda e Marketing, Brasil Rossana Reguillo, Instituto de Estudos Superiores do Ocidente, Mexico Rousiley Celi Moreira Maia, Universidade Federal de Minas Gerais, Brasil Sebastião Carlos de Morais Squirra, Universidade Metodista de São Paulo, Brasil Sebastião Guilherme Albano da Costa, Universidade Federal do Rio Grande do Norte, Brasil

Simone Maria Andrade Pereira de Sá, Universidade Federal Fluminense, Brasi Tiago Quiroga Fausto Neto, Universidade de Brasília, Brasil Suzete Venturelli, Universidade de Brasília, Brasil Valério Cruz Brittos, Universidade do Vale do Rio dos Sinos, Brasil Valerio Fuenzalida Fernández, Puc-Chile, Chile Veneza Mayora Ronsini, Universidade Federal de Santa Maria, Brasil Vera Regina Veiga França, Universidade Federal de Minas Gerais, Brasil Valerio Fuenzalida Fernández, Puc-Chile, Chile Veneza Mayora Ronsini, Universidade Federal de Santa Maria, Brasil Vera Regina Veiga França, Universidade Federal de Minas Gerais, Brasil

\section{COMISSÃO EDITORIAL}

Adriana Braga I Pontifícia Universidade Católica do Rio de Janeiro, Brasil Felipe Costa Trotta I Universidade Federal de Pernambuco, Brasi

CONSULTORES AD HOC

Édison Gastaldo, Universidade Federal Rural do Rio de Janeiro, Brasil

EDIÇÃO DE TEXTO E RESUMOS I Susane Barros

SECRETÁRIA EXECUTIVA I Juliana Depiné

EDITORAÇÃO ELETRÔNICA I Roka Estúdi

TRADUÇÃo I Sieni Campos e Robert Finnegan
COMPós I www.compos.org.br

Associação Nacional dos Programas de Pós-Graduação em Comunicação

Presidente

Julio Pinto

Pontifícia Universidade Católica de Minas Gerais, Brasi

juliopinto@pucminas.br

Vice-presidente

Itania Maria Mota Gomes

Universidade Federal da Bahia, Brasi

itania@ufba.br

Secretária-Geral

Inês Vitorino

Universidade Federal do Ceará, Brasil

inesvict@gmail.com 